\title{
Non-uniform electrodeposition of zinc on the (0001) plane
}

Takato Mitsuhashi ${ }^{1}$, Yasumasa Ito ${ }^{2,3,{ }^{*}}$, Yukihisa Takeuchi ${ }^{1}$, Shunta Harada ${ }^{1}$ and Toru Ujihara ${ }^{1,3}$ ${ }^{1}$ Department of Materials Science and Engineering, Graduate School of Engineering, Nagoya University, Furo-cho, Chikusa-ku, Nagoya, Aichi 464-8603, Japan

2Department of Mechanical Science and Engineering, Graduate School of Engineering, Nagoya University, Furo-cho, Chikusa-ku, Nagoya, Aichi 464-8603, Japan

${ }^{3}$ Green Mobility Collaborative Research Center, Nagoya University, Furo-cho, Chikusa-ku, Nagoya, Aichi 464-8603, Japan

Abstract: Transition of zinc morphology and crystallographic orientations with increasing charge capacity is examined to identify the origin of non-uniform electrodeposition. Galvanostatic charge experiments for zinc are carried out for both polycrystalline and oriented zinc substrates to investigate the effects of electrodeposited zinc on electrodepositing zinc. The results show that the crystallographic orientations of the zinc substrates strongly affect the morphology of the electrodeposited zinc. Non-uniformity appears in the (0001)-oriented case prior to the polycrystalline, (1010)- and (2 $\overline{1} \overline{1} 0)$-oriented cases. The (0001) plane initiates non-uniform zinc electrodeposition even in the polycrystalline case. It is attributed to the high density and low diffusivity of the ad-atom on the wide terrace structure. It is also indicated that porous structure appears from aggregated hexagonal hillocks with random orientations which initially are oriented hexagonal hillocks.

\section{*Corresponding Author}

Name: Yasumasa Ito

Address: Department of Mechanical Science and Engineering, Graduate School of Engineering, Nagoya University, Furo-cho, Chikusa-ku, Nagoya, Aichi 464-8603, Japan

Telephone number: $\quad+81-52-789-4488$

e-mail address: yito@nagoya-u.jp 


\section{Body of paper.}

\section{Introduction}

Metal-anode rechargeable batteries such as lithium and zinc have been receiving increasing attentions as next-generation batteries. Lithium has a high theoretical specific capacity of $3860 \mathrm{mAh} \mathrm{g}^{-1}[1]$. This advantage is maximized by developing metal-lithium batteries for mobile uses such as portable electronic devices and electric vehicles. On the other hand, zinc has advantages such as low cost, abundant supply, non-toxicity, safety, and scalability [2,3]. Therefore, zinc-anode batteries are perfect for large and stationary uses for smart-grid, peak-shaving, load-leveling, etc. Despite these high expectations for wide variety of applications, metal-anode rechargeable batteries have not been widely spread yet [3-6]. This is mainly due to short cycle life due to short circuit which is caused by non-uniform electrodeposition of metal anodes on charging [7-8].

Since it is a classical problem, a number of methods have been proposed and explored to suppress the non-uniformity. In case of zinc, typical methods are the use of additives in electrode or electrolyte [9-13], flowing electrolyte [7,12], pulse charging [14], and optimization of substrates [15-18]. Here electrodeposition process of a metal generally consists of the following three steps: (1) diffusion of the metal ion $\left(\mathrm{M}^{\mathrm{Z}+}\right)$ to an electrode surface in electrolyte, (2) generation of an adatom $\left(\mathrm{M}_{\mathrm{ad}}\right)$ by a charge transfer reaction, and (3) surface diffusion of the adatom and crystallization $\left(\mathrm{M}_{\mathrm{ad}} \rightarrow \mathrm{M}_{\text {crystal }}\right)$. The flowing electrolyte and pulse charging methods mainly target the step (1) through the control of the metal ion concentration at the electrode surface and current density, respectively. The effects of additives vary depending on the species. The optimization of substrates is aimed at controlling the step (3). Although past studies have shown that materials of substrates influence on metal electrodeposition [17], considering that metal electrodeposition is a crystal growth driven electrochemically, it is important to approach not only from a material point of view but also from a crystal point of view [19]. Actually McBreen et al. have investigated effects of substrates on zinc electrodeposition [15-17] and showed that mismatch of crystallographic orientations between zinc and the substrates significantly influences on the morphology. In cases of gold, copper, cadmium, and zinc substrates, the difference of the basal plane from zinc is small and electrodeposited zinc is oriented parallel to the basal plane. On the other hand, in the cases of lead and indium substrates, the difference is large and electrodeposited zinc is oriented perpendicular to the basal plane [17]. Recent study by Desai et al. [20] showed that compact zinc deposited on brass is shown to have $\sim 60 \%$ 
(112̄2) preferred orientation.

However, the importance of crystallographic orientation lies not only in the relationship between the substrate and depositing metal but also in the relationship between the deposited and depositing metal anode since, in practice, it is required to retain the compact and uniform deposition with preferable morphology as long as possible to realize metal anodes with a long cycle life and high energy density. In this respect, it is of great interest to clarify transitions of its morphology and crystallographic orientations with increasing charge capacity, particularly in terms of identifying the origin of non-uniformity [21]. We, therefore, carried out galvanostatic charge experiments for zinc. Polycrystalline and oriented zinc are employed as substrates of the working electrode to clearly reveal the effects of crystallographic orientations on transitions of the morphology.

\section{Experimental details}

\section{2-1. Preparation of the oriented zinc substrate}

The oriented zinc crystal as the substrate for zinc electrode was grown by the Bridgman method in our laboratory at Nagoya University. Fig. 1 shows the schematic image of crystal growth furnace. Zinc grain with a purity of $>99.99 \%$ and a diameter of 3-7 mm (Japan Metal Services) was used as the original material. After being ultrasonically cleaned in acetone, methanol, and ultrapure water whose electric resistance is $18 \mathrm{M} \Omega \cdot \mathrm{cm}$, the oxide layers on the zinc grains were etched with hydrochloric acid and water solution (50/50). After the process, they were vacuum-sealed in a quartz tube to prevent from oxidation. The furnace temperature was initially raised above the melting point $(693 \mathrm{~K})$ and then lowered at a rate of $6 \mathrm{~K} \mathrm{~min}^{-1}$ down to the room temperature $(298 \mathrm{~K})$. The vertical temperature gradient was kept at 15 $\mathrm{K} \mathrm{cm}^{-1}$ around the tube. Fig. 2 is a photo image of the grown zinc crystal. In this study, we used two different surfaces of the zinc (named as Surfaces I and II in Fig. 2) as the electrode substrates. The surfaces were mechanically polished with 1.0 and $0.3 \mu \mathrm{m}$ aluminum suspensions (Baikalox) for 10 minutes after grinding with the 1000\#, 2000\# (Maruto), and 4000\# (Struers) SiC waterproof abrasive papers. Note that the grown zinc was carefully washed with deionized water to remove residual abrasive media at each process.

The crystallographic characterization was carried out by an electron backscatter diffraction (EBSD) method (PHI 700 SN). The obtained EBSD patterns were analyzed by means of the orientation imaging microscopy $\left(\mathrm{OIM}^{\mathrm{TM}}\right)$ system. Fig. 3 shows the 
analyzed crystallographic characteristics and Scanning-Electron-Microscopy (SEM) images of the Surfaces I and II, together with crystalline structure of zinc. Since zinc has a hexagonal structure, there are three possible crystallographic orientations: (0001), $(10 \overline{1} 0)$ and ( $2 \overline{1} \overline{1} 0)$. Fig. 3 illustrates that the Surface I is mainly (10 $\overline{1} 0)$-oriented but the $(2 \overline{1} \overline{1} 0)$ plane is partially inserted while the Surface II is mostly (0001)-oriented for the entire area.

\section{2-2 Galvanostatic charge experiments}

Fig. 4 shows the battery cell used for the galvanostatic charge experiments. The active surface area for electrochemical reaction is $5.0 \mathrm{~mm} \times 0.5 \mathrm{~mm}$ for both the anode (working electrode) and cathode and the electrodes were set with a $3.0 \mathrm{~mm}$ gap. The current collector for the cathode was a nickel sheet with a purity of $>99.99 \%$ (Japan Metal Services) meaning oxygen evolves on charge. Note that the gap between the anode and cathode is large and zinc electrodeposition is unaffected by the cathode reaction. For the working electrode, zinc crystal as the substrate was welded to the tip of a cupper current collector by conductive silver paste (Fujikura Kasei D-550). The current collector and substrate were wrapped with a polytetrafluoroethylene heat shrink tube to prevent zinc electrodeposition on the sides of the working electrode. As the zinc substrate, both the polycrystalline and oriented zinc crystals were used. The details of the lab-made oriented zinc substrates are already described in Section 2-1. On the other hand, the polycrystalline zinc substrate has a purity of 99.99\% (Japan Metal Services) and its grain size is typically of the order of $10 \mu \mathrm{m}$. It was prepared by polishing in the same way as for the oriented crystal. The electrolyte was prepared by dissolving potassium hydroxide (KOH) (JIS grade, Nacalai tesque) at a concentration of $45 \mathrm{wt} \%$ into DI water. Zinc oxide powder (Special grade, Kishida chemical) as the anode material was initially dissolved into the electrolyte at a concentration of $0.62 \mathrm{~mol} \mathrm{~L}^{-1}$ which corresponds to a fractional saturation of about $30 \%$. The casing of the battery cell was made of acrylic.

Galvanostatic (constant current) charge was carried out using an electrochemical measurement system (Hokuto Denko HZ-7000). Table 1 lists the experimental conditions. The current density was set to 10 and $50 \mathrm{~mA} \mathrm{~cm}^{-2}$ and the charge capacity was set to $0.1,1.0,5.0,7.5$, and $10 \mathrm{mAh} \mathrm{cm}^{-2}$. Hereinafter, experimental conditions are

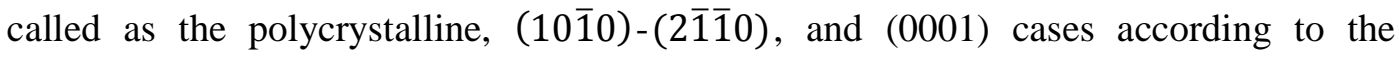
crystallographic orientation of the substrates. The surface morphology of zinc was examined by a SEM (Hitachi S4300). The acceleration voltage and emission current were set to $10 \mathrm{kV}$ and $10 \mu \mathrm{A}$, respectively. Surface roughness of the electrodeposited 
zinc was measured by a laser microscope (Keyence VK-X100). Note that the charged working electrodes were soaked into DI water to remove residual $\mathrm{KOH}$ solution and washed in acetone several times to dehydrate and prevent from oxidation before the inspections.

\section{Results}

Fig. 5 shows the SEM images of the working electrode for a current density of 50 $\mathrm{mA} \mathrm{cm}$ and a charge capacity of $1.0 \mathrm{mAh} \mathrm{cm}^{-2}$. It is clearly observed that the crystallographic orientation of the substrates significantly affects the morphology of electrodeposited zinc. In the polycrystalline case (Fig. 5 (a)), it has a layered structure but two layering directions are mixed. In the (10 $\overline{1} 0)-(2 \overline{1} \overline{1} 0)$ case (Fig. 5(b)), on the other hand, sheet-like structure with a consistent direction (indicated by solid line) is dominant but ridge-like structure (indicated by dash line) is also observed. By comparison with the SEM images in Fig.3, the latter structure is expected to be the ( $2 \overline{1} \overline{1} 0)$ plane of zinc crystal. In addition, the sheet-like area is much larger than the ridge-like area. It is the same order as that of (10 $\overline{1} 0)$ - and (2 $\overline{1} \overline{1} 0)$-oriented planes for the (10 $\overline{1} 0)-(2 \overline{1} \overline{1} 0)$ substrate. Thus it is suggested that zinc deposits epitaxially and the sheet- and ridge-like structures correspond to (10 $\overline{1} 0)$ and $(2 \overline{1} \overline{1} 0)$ planes, respectively. In the (0001) case (Fig. 5(c)), a number of flake-like zinc crystals are randomly precipitated on a flat and uniform surface. The EBSD analysis revealed that the flat surface is the (0001) plane, meaning that zinc initially electrodeposited epitaxially. The flakes have almost the same shape with sizes of maximum $5 \mu \mathrm{m}$ and seem to have the ( $2 \overline{1} \overline{1} 0$ ) facet plane, though it was impossible to analyze by the EBSD.

Fig. 5 illustrates that the surface is relatively flat and uniform in the polycrystalline and $(10 \overline{1} 0)-(2 \overline{1} \overline{1} 0)$ cases, whereas it is not uniform in the (0001) case. As mentioned in the Introduction, surface uniformity is a critical factor for battery performance. To quantitatively evaluate the surface roughness, the height fluctuation was examined by the laser microscope. Fig. 6 shows the results in a form of a color contour map and their quantitative evaluation is summarized in Table 2. It shows that the deposition on the (1010) plane has the lowest arithmetic average roughness $\left(R_{\mathrm{a}}\right)$ of $0.092 \mu \mathrm{m}$, while that on the polycrystalline and $(2 \overline{1} \overline{1} 0)$ planes is about $0.27 \mu \mathrm{m}$. On the other hand, the height fluctuation in the (0001) case is about 6 times as much as that on the (1010) substrate and twice as much as that on the $(2 \overline{1} \overline{1} 0)$ and polycrystalline substrates because of the flake-like precipitation, which would initiate non-uniformity on the (0001) plane earlier than the other cases.

Zinc morphology in the polycrystalline and (0001) cases was examined for a current 
density of $10 \mathrm{~mA} \mathrm{~cm}^{-2}$. Fig. 7 shows the SEM images of the working electrode for a charge capacity density of $1.0 \mathrm{mAh} \mathrm{cm}^{-2}$. The morphology in the polycrystalline case (Fig. 7(a)) is relatively uniform and similar to that for the current density of $50 \mathrm{~mA} \mathrm{~cm}^{-2}$. In the (0001) case (Fig. 7(b)), on the other hand, random spotty depositions due to 2D-nucleations on step-terrace structures are observed. The average width of the terrace is $10 \mu \mathrm{m}$ and it is (0001)-oriented. With respect to the spotty depositions, the one surrounded by the solid line has an oriented hexagonal hillocks structure (Fig. 7(c)) and the other one surrounded by the dash line has an aggregated hexagonal hillocks structure with random orientations i.e. polycrystalline structure (Fig. 7(d)). In addition, although it is not shown in Fig. 7(b), porous zinc deposition with partially hexagonal structures was also observed in this case, as in Fig 7(e). This porous structure is usually observed at low current density experiments in a number of past studies. 2, 7, 12,13 These images suggest that the porous structures appear from the aggregated hexagonal hillocks with random orientations which initially are oriented hexagonal hillocks. The dependency of the crystalline structure of the initial zinc deposition on the current densities is explained in the past studies [22-25]. Pangarov [25] investigated the dependency of nucleation for metals which have a hexagonal close-packed (hcp) structure on equilibrium. He concluded that the preferential crystallographic orientation of the electrodeposited metal is (0001) and (1011) when the system is closer to equilibrium, it is (2 $\overline{1} \overline{1} 0)$ when the system is moderately far to equilibrium, and it is $(10 \overline{1} 0)$ and $(11 \overline{2} 2)$ when the system is far to equilibrium. In the present study, on the other hand, the (0001) plane appears at a current density of $10 \mathrm{mAh} \mathrm{cm}^{-2}$ (Fig. 7(b)) and

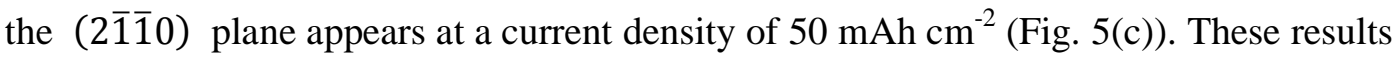
are consistent with his study.

Regardless of current density, non-uniform zinc precipitation was observed in the (0001) case for a charge capacity of $1.0 \mathrm{mAh} \mathrm{cm}^{-2}$, whereas such non-uniform deposition is not observed in the polycrystalline and (10 $\overline{1} 0)-(2 \overline{1} \overline{1} 0)$ cases. This means that the (0001) plane initiates non-uniformity prior to the other planes. Since the polycrystalline substrate is supposed to include the (0001) plane, the morphology transition is further investigated by increasing charge capacity to find out whether the non-uniform electrodeposition provokes on the (0001) plane. Fig. 8 shows the SEM images in the polycrystalline case for charge capacities of (a)5.0 $\mathrm{mAh} \mathrm{cm}^{-2}$, (b)7.5 mAh $\mathrm{cm}^{-2}$, and (c)10 $\mathrm{mAh} \mathrm{cm}^{-2}$. Spotty depositions, pointed by arrows in Fig. 8(a), are observed at the charge capacity of $5.0 \mathrm{mAh} \mathrm{cm}^{-2}$. Many of these are flat and hexagonal, indicating that their facet is the (0001) plane. On the other hand, porous structure is randomly observed in the case of $7.5 \mathrm{mAh} \mathrm{cm}^{-2}$ and the area becomes larger at the 
charge capacity of $10 \mathrm{mAh} \mathrm{cm}^{-2}$. This sequence is basically the same as that in the (0001) case and it is again shown that the (0001) plane initiates non-uniform zinc electrodeposition.

\section{Discussion}

The mechanism that the non-uniform electrodepostion tends to appear on the (0001) plane is explained as follows. Fig. 9 illustrates the deposition process of zinc in the (0001) case. The key is the transition from (b) to (c). The (0001)-oriented wide terrace is a habit plane for zinc whose crystal structure is hexagonal, and in general, 2D-nucleation easily occurs on such a smooth surface because of the high ad-atom density. Moreover, the activation energy of surface diffusion for the (0001) plane is 6.8

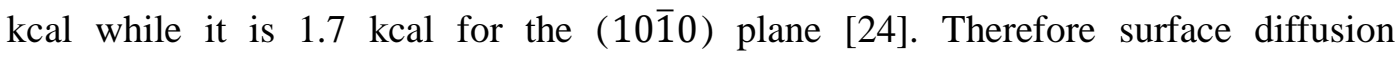
coefficient on the (0001) plane is smaller than other planes. The lower diffusivity prevents the incorporation of the ad-atoms at step edges which plays a role of a sink of ad-atoms. Therefore, non-uniform electrodeposition is most likely to take place on the (0001) plane.

In the case of the other surfaces, on the other hand, the 2D-nucleation hardly occurs because of the absence of the wide terrace structure, though formation of the hexagonal platelets, whose main crystallographic orientation of the facet is (0001), eventually takes place for some reason. Once it happened, the non-uniform electrodeposition precipitates on platelets, which is the same process as the precipitations in the case of the (0001)-oriented substrate.

\section{Conclusions}

Galvanostatic charge experiments for zinc are carried out for both polycrystalline and oriented zinc substrates to identify the origin of non-uniformity by clarifying transitions of zinc morphology and crystallographic orientations. The results clearly show that the crystallographic orientation of the zinc substrate strongly affects the morphology of electrodeposited zinc. In particular, the (0001)-oriented substrate initiates

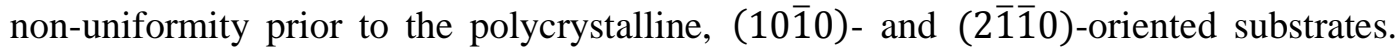
This earlier appearance of non-uniformity on the (0001) plane is attributed to the high density and low diffusivity of the ad-atom on the wide terrace structure. It is also indicated that porous structure appears from aggregated hexagonal hillocks with random orientations which initially are oriented hexagonal hillocks.

The present study suggests that, to suppress non-uniform electrodeposition of zinc, it 
is important to select a current collector whose the activation energy of surface diffusion is small, which delays 2D-nucleation.. Moreover, it is preferable that the surface is the smooth facet plane to avoid current concentration. Since the (1010) plane has the highest diffusivity for zinc and tends to layer laterally rather than vertically, a current collector that allows (1010)-orientation is desirable to keep uniform and compact electrodeposition.

\section{Acknowledgements}

Part of this study was supported by the Adaptable \& Seamless Technology Transfer Program through Target-driven R\&D (A-STEP) by the Japan Science and Technology Agency (JST). 


\section{References.}

1. S. Liu, J. Yang, L. Yin, Z. Li, J. Wang, Y., Nuli, Lithium-rich Li 2.6BMg 0.05 alloy as an alternative anode to metallic lithium for rechargeable lithium batteries, Electrochim Acta 56 (2011) 8900-8905.

2. Y. Ito, X. Wei, , D. Desai, D. Steingart, S. Banerjee, An indicator of zinc morphology transition in alkaline electrolyte, J. Power Sources 211 (2012) 119-128.

3. D. Turney, M. Shmukler, J. Galloway, M. Klein, Y. Ito, M. Nyce T. Sholklapper, S. Banerjee, Development and testing of an economic grid-scale flow-assisted zinc/nickel-hydroxide alkaline battery, J. Power Sources, 264 (2014) 49-58.

4. F.R. Mclarnon, E.J. Cairns, The secondary alkaline zinc electrode, J. Electrochem. Soc. 138 (1991) 645-656.

5. S. Liu, H. Wang, N. Imanishi, T. Zgang, A. Hirano, Y. Takeda, O. Yamamoto, J. Yang, Effect of co-doping nano-silica filler and $\mathrm{N}$-methyl-N-propylpiperidinium bis (trifluoromethanesulfonyl)imide into polymer electrolyte on Li dendrite formation in Li/poly(ethylene oxide)-Li(CF3SO2)2N/Li, J. Power Sources, 196 (2011) 7681-7686.

6. T.D. Gregory, R.J. Hoffman, R.C. Winterton, Nonaqueous electrochemistry of magnesium, J. Electrochem. Soc., 137 (1990) 775-780. 
7. Y. Ito, M. Nyce, R. Plivelich, M. Klein, D. Steingart, S. Banerjee, Zinc morphology in zinc-nickel flow assisted batteries and impact on performance, J. Power Sources 196 (2011) 2340-2345.

8. K. Nishikawa, T. Mori, T. Nishida, Y. Fukunaka, M. Rosso, Li dendrite growth and Li+ ionic mass transfer phenomenon, J. Electroanal. Chem. 661 (2011) 84-89.

9. C.W. Lee, K. Sathiyanarayanan, S.W. Eoma, H.S. Kima,M.S. Yun, Novel electrochemical behavior of zinc anodes in zinc/air batteries in the presence of additives, J. Power Sources 159 (2006) 1474-1477.

10. R. Shivkumar, G.P. Kalaignan, T.J. Vasudevan, Effect of additives on zinc electrodes in alkaline battery systems, J. Power Sources 55 (1995) 53-62.

11. J.L. Zhu, Y.H. Zhou, C.Q. Gao, Influence of surfactants on electrochemical behavior of zinc electrodes in alkaline solution, J. Power Sources 72 (1998) 231-235.

12. Y. Sato, M. Kanda, H. Niki, M. Ueno, K. Murata, T. Shirogami, T. Takamura, Long life sealed nickel-zinc cell using a new separator, J. Power Sources 9 (1983) 147-159.

13. G. Adzic, J. McBreen, M. Chu, Adsorption and alloy formation of zinc layers on silver, J. Electrochem. Soc. 128 (1981) 1691-1697. 
14. M.G. Chu, J. McBreen, G. Adzic, Substrate effects on zinc deposition from zincate solutions. I . Deposition on Cu, Au, Cd and Zn, J. Electrochem. Soc. 128 (1981) 2281-2286.

15. J. McBreen, M.G. Chu, G. Adzic, Substrate effects on zinc deposition from zincate solutions: II . Deposition on Pb, Tl, Sn, and In, J. Electrochem. Soc. 128 (1981) $2287-2292$.

16. L. Zhang, J. Cheng, Y.S. Yang, Y.H. Wen, X.D. Wang, G.P. Cao, Study of zinc electrodes for single flow zinc/nickel battery application, J. Power Sources. 179 (2008) 381-387.

17. R.A. Oriani, C.J. Johnson, Modern Aspects of Electrochemistry, No. 5, J. O’M. Bockris (ed.) Prenum Press, New York (1969).

18. J.P. Hirth, G.M. Pound, Condensation and Evaporation, Vol. 2, in the series Progress in Materials Science, B. Chalmer (ed.) Macmillan Co., New York (1963).

19. D. Desai, X. Wei, D. Steingart, S. Banerjee, Electrodeposition of preferentially oriented zinc for flow-assisted alkaline batteries, J. Power Sources 256 (2014) 145-152. 
20. H. Wang, S. Zhou, C. Chen, Q. Wang, Electrochemical performance of Zn(002) and $\mathrm{Zn}(100)$ single crystals in 6.0mol.L-1 KOH, Chinese J. Chem. Eng. 14 (2006) $551-554$.

21. Y. Wen, T. Wang, J. Cheng, J. Pan, G. Cao, Y. Yang, Lead ion and tetrabutylammonium bromide as inhibitors of the growth of spongy zinc in single flow zinc/nickel batteries, Electrochem. Acta 59 (2012) 64-68.

22. L. Guo, P.C. Searson, On the influence of the nucleation overpotential on island growth in electrodeposition, Electrochm. Acta 55 (2010) 4086-4091.

23. Y.H. Wen, J. Cheng, L., Zhang, X., Yan, Y.S., Yang, The inhibition of the spongy electrocrystallization of zinc from doped flowing alkaline zincate solutions, J. Power Sources. 193 (2009) 890-894.

24. S. Haruyama, A theoretical study on metal electrodeposition reactions, J. Electrochem. Soc. Jpn., 31 (1963) 478-484. (in Japanese)

25. N.A. Pangarov, Preferred orientations in electro-deposited metals, J. Electroanal. Chem. 9 (1964) 70-85. 


\section{List of Figures}

Fig. 1. Schematic image of the crystal growth furnace.

Fig. 2. Grown zinc crystal.

Fig. 3. Crystallographic characteristics(a) and SEM images(b) of the Surfaces I and II.

Fig. 4. Battery cell. (a) Photo image; (b) electrodes configuration.

Fig. 5. SEM images of the working electrode. (a) polycrystalline (Test \#2) case; (b)

(10 $\overline{1} 0)-(2 \overline{1} \overline{1} 0)$ case (Test \#4); (c) (0001) case (Test \#6). The current density is 50 $\mathrm{mA} \mathrm{cm}{ }^{-2}$ and the charge capacity is $1.0 \mathrm{mAh} \mathrm{cm}^{-2}$.

Fig. 6. Surface roughness of the electrodeposited zinc. (a) polycrystalline case (Test \#2);

(b) $(10 \overline{1} 0)-(2 \overline{1} \overline{1} 0)$ case (Test \#4); (c) (0001) (Test \#6). The current density is 50 $\mathrm{mA} \mathrm{cm}{ }^{-2}$ and the charging capacity density is $1.0 \mathrm{mAh} \mathrm{cm}$.

Fig. 7. SEM images of the working electrode. (a) polycrystalline case (Test \#9); (b)-(e)

(0001) case (Test \#14). The current density is $10 \mathrm{~mA} \mathrm{~cm}^{-2}$ and the charge capacity is $1.0 \mathrm{mAh} \mathrm{cm}{ }^{-2}$. 
Fig. 8. SEM images of the working electrode in the polycrystalline case. The current density is $10 \mathrm{~mA} \mathrm{~cm}^{-2}$. The charge capacity is (a) $5.0 \mathrm{mAh} \mathrm{cm}^{-2}$ (Test \#10) and (b) 7.5 $\mathrm{mAh} \mathrm{cm}{ }^{-2}$ (Test \#11), and (c) $10.0 \mathrm{mAh} \mathrm{cm}^{-2}$ (Test \#12), respectively.

Fig. 9. Formation process of non-uniform electrodeposition in the (0001) case: (a) At the early stage of charge, layer-by-layer growth proceeds with smooth surface while maintaining step-terrace structure; (b) wide terraces are formed due to step bunching; (c) spotty structures form via recurring 2D-nucleations on the wide terraces and the current concentration at the ledges results in the preferential formation of electrodeposition; and (d) the electrodeposition enhance more precipitation, and finally, the porous non-uniform electrodeposition forms. 


\section{List of Tables}

Table 1. Experimental conditions.

Table 2. Arithmetic average roughness $\left(R_{\mathrm{a}}\right)$ at charge capacity density of $1.0 \mathrm{mAh} \mathrm{cm}$. 
Tables.

Table 1. Experimental conditions.

\begin{tabular}{crcrr}
\hline Test & $\begin{array}{c}\text { Current } \\
\text { density } \\
{\left[\mathrm{mA} \mathrm{cm}^{-2}\right]}\end{array}$ & $\begin{array}{c}\text { Crystallographic } \\
\text { orientation of the } \\
\text { current collector }\end{array}$ & $\begin{array}{c}\text { Charge capacity } \\
{\left[\mathrm{mAh} \mathrm{cm}^{-2}\right]}\end{array}$ & $\begin{array}{c}\text { Charge time } \\
{[\mathrm{sec}]}\end{array}$ \\
\hline$\# 1$ & 50 & Polycrystalline & 0.1 & 7.2 \\
$\# 2$ & 50 & Polycrystalline & 1.0 & 72 \\
$\# 3$ & 50 & Polycrystalline & 10.0 & 720 \\
$\# 4$ & 50 & $(10 \overline{1} 0)-(2 \overline{1} \overline{1} 0)$ & 1.0 & 72 \\
$\# 5$ & 50 & $(0001)$ & 0.1 & 7.2 \\
$\# 6$ & 50 & $(0001)$ & 1.0 & 72 \\
$\# 7$ & 50 & $(0001)$ & 10.0 & 720 \\
$\# 8$ & 10 & Polycrystalline & 0.1 & 36 \\
$\# 9$ & 10 & Polycrystalline & 1.0 & 360 \\
$\# 10$ & 10 & Polycrystalline & 5.0 & 1800 \\
$\# 11$ & 10 & Polycrystalline & 7.5 & 2700 \\
$\# 12$ & 10 & Polycrystalline & 10 & 3600 \\
$\# 13$ & 10 & $(0001)$ & 0.1 & 36 \\
$\# 14$ & 10 & $(0001)$ & 1.0 & 3600 \\
$\# 15$ & 10 & $(0001)$ & 10.0 & \\
\hline
\end{tabular}

Table 2. Arithmetic average roughness $\left(R_{\mathrm{a}}\right)$ at charge capacity density of $1.0 \mathrm{mAh}$ $\mathrm{cm}^{-2}$.

\begin{tabular}{cc} 
Current collector orientation & $R_{\mathrm{a}}[\mu \mathrm{m}]$ \\
\hline Polycrystalline & 0.273 \\
$(10 \overline{1} 0)$ & 0.092 \\
$(2 \overline{1} \overline{1} 0)$ & 0.262 \\
$(0001)$ & 0.547 \\
\hline
\end{tabular}


Figures

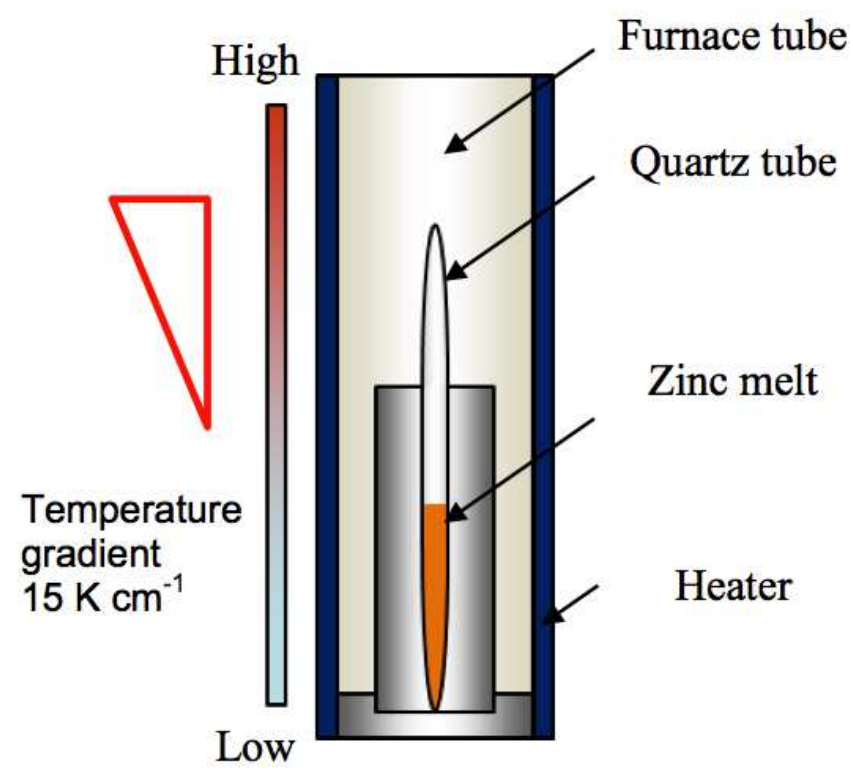

Fig. 1. Schematic image of the crystal growth furnace. 


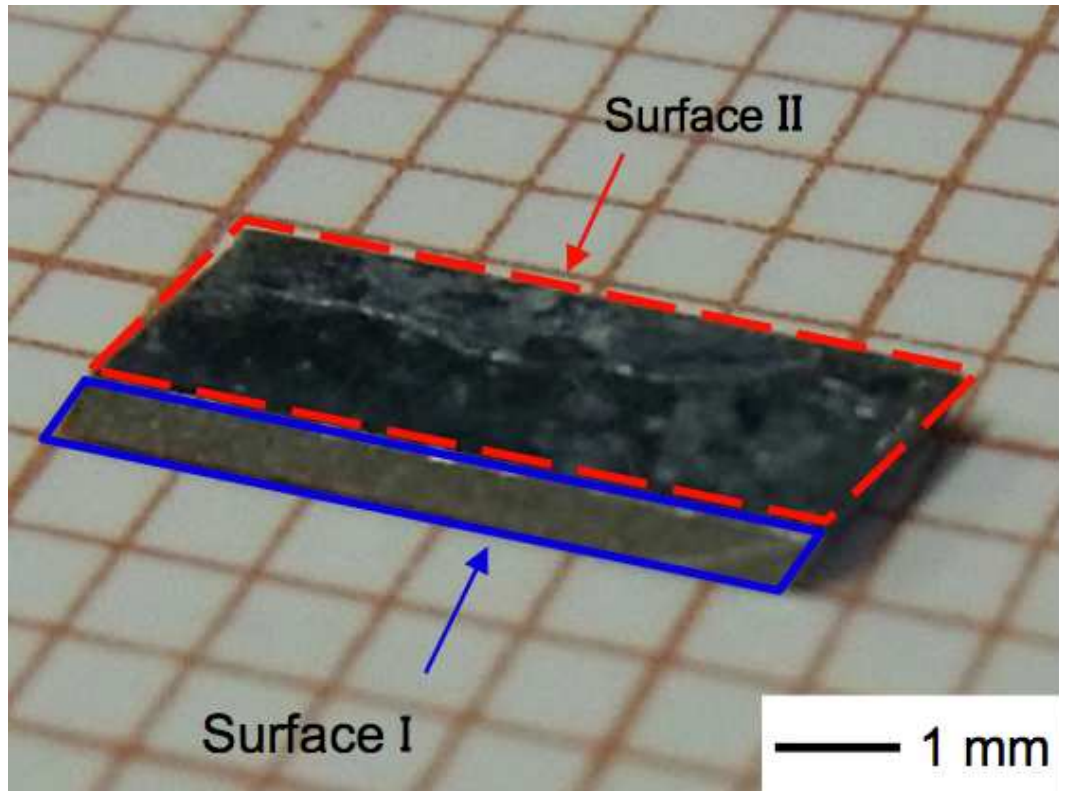

Fig. 2. Grown zinc crystal. 

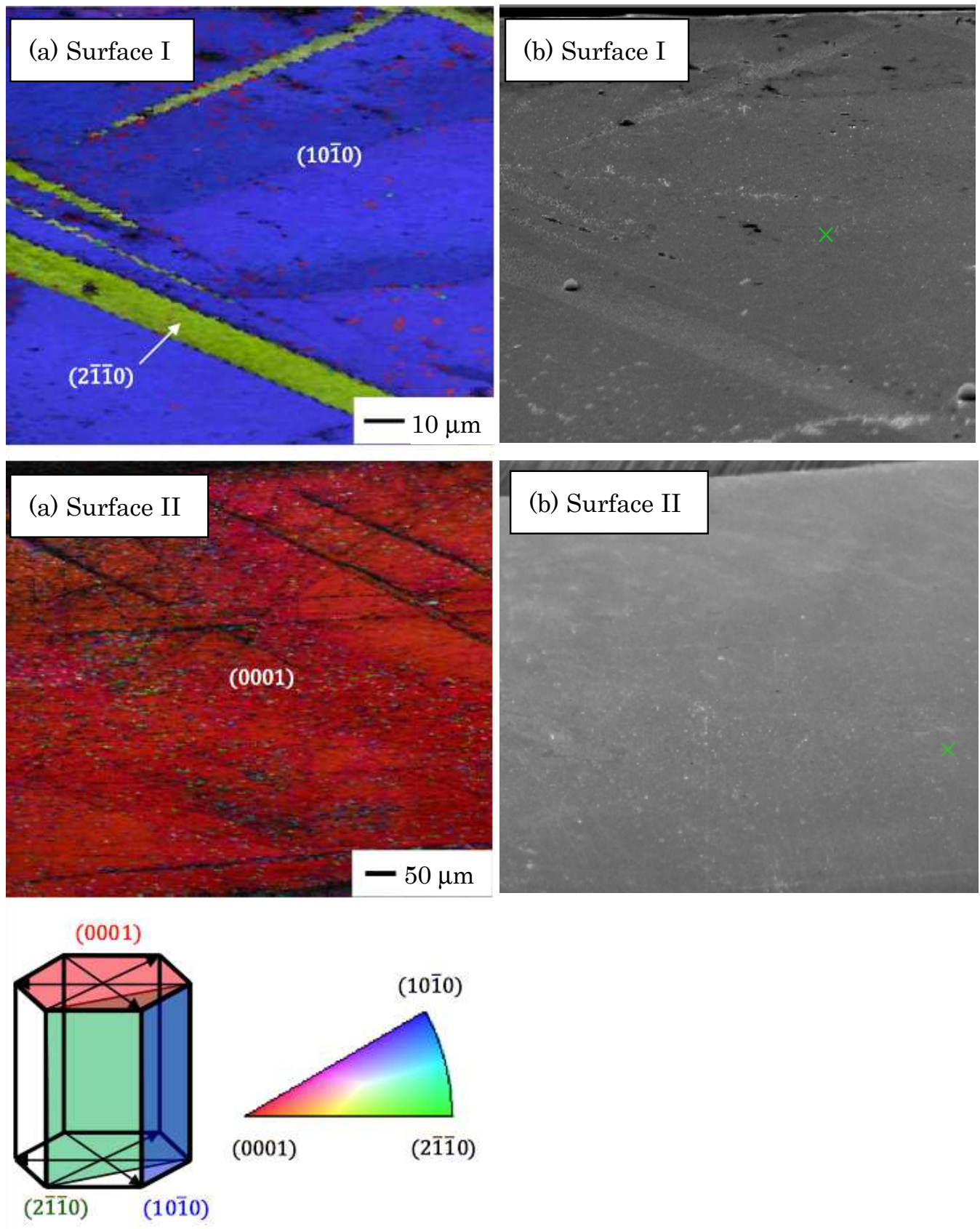

Fig. 3. Crystallographic characteristics(a) and SEM images(b) of the Surfaces I and II. 

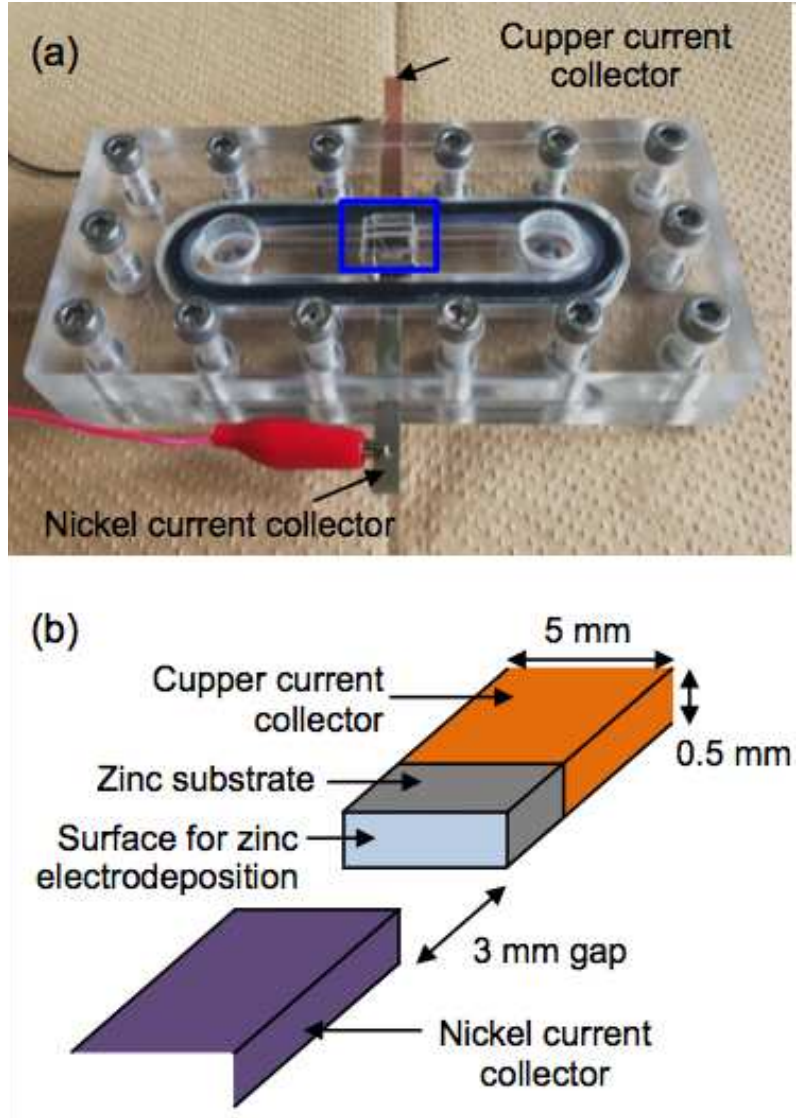

Fig. 4. Battery cell. (a) Photo image; (b) electrodes configuration. 

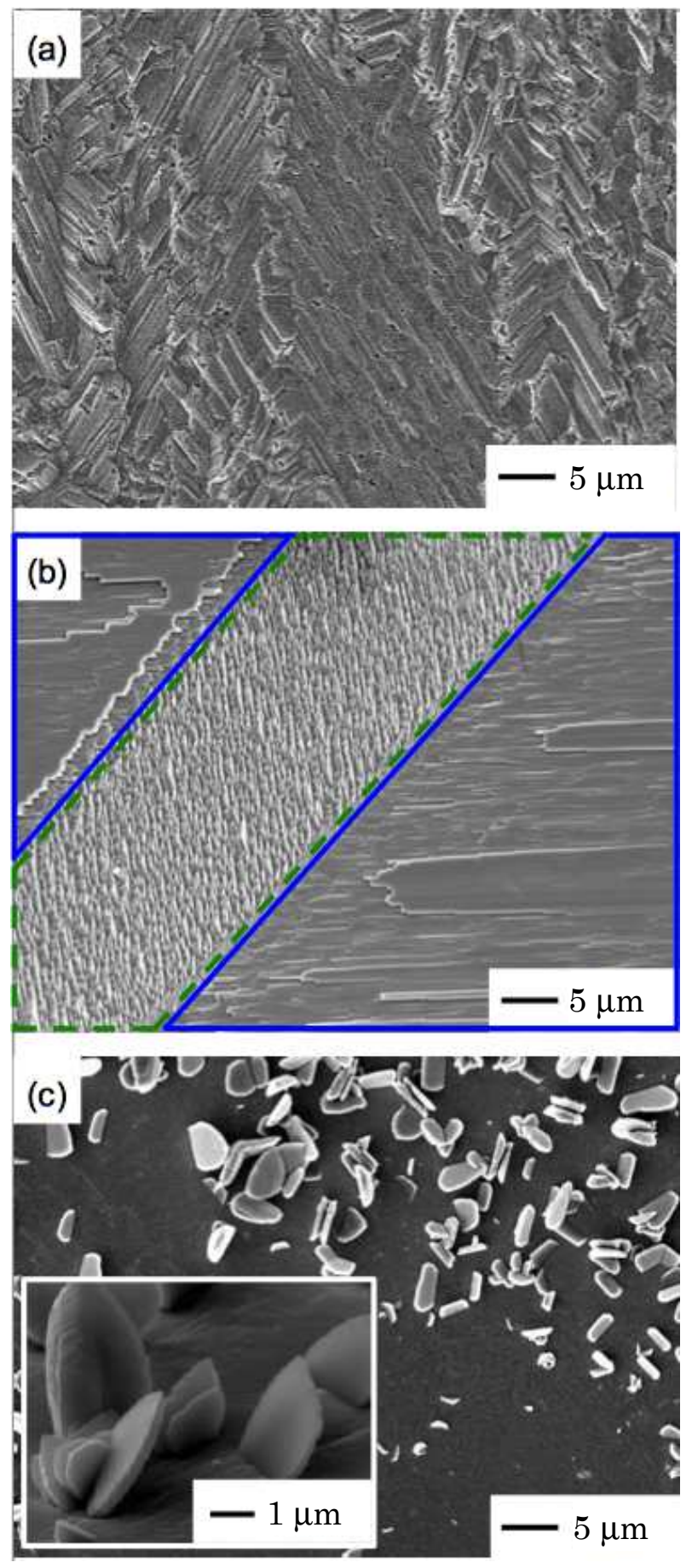

Fig. 5. SEM images of the working electrode. (a) polycrystalline (Test \#2) case; (b) (10 $\overline{1} 0)-(2 \overline{1} \overline{1} 0)$ case (Test \#4); (c) (0001) case (Test \#6). The current density is 50 $\mathrm{mA} \mathrm{cm}{ }^{-2}$ and the charge capacity is $1.0 \mathrm{mAh} \mathrm{cm}^{-2}$. 

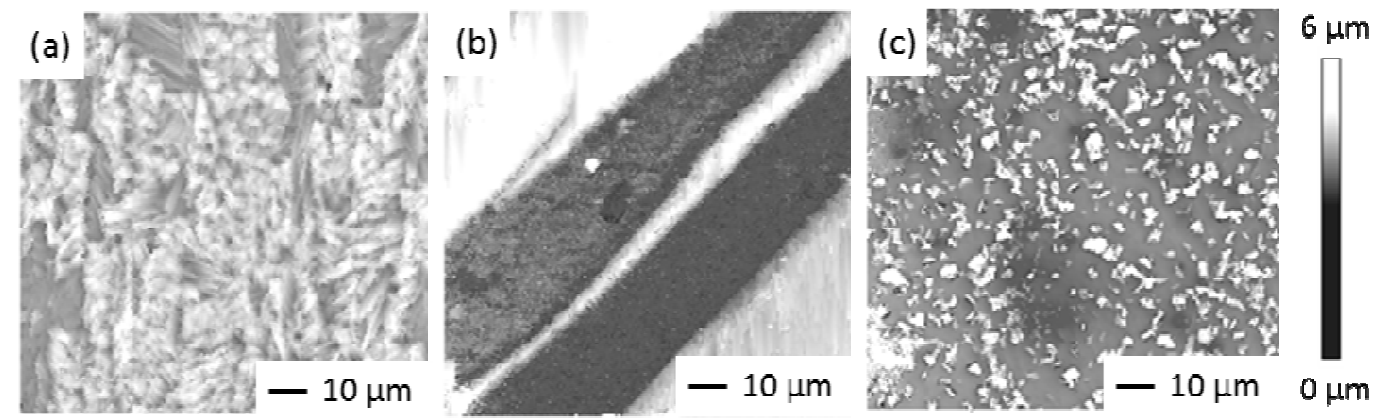

Fig. 6. Surface roughness of the electrodeposited zinc. (a) polycrystalline case (Test \#2); (b) $(10 \overline{1} 0)-(2 \overline{1} \overline{1} 0)$ case (Test \#4); (c) (0001) (Test \#6). The current density is 50 $\mathrm{mA} \mathrm{cm}$ and the charging capacity density is $1.0 \mathrm{mAh} \mathrm{cm}^{-2}$. 

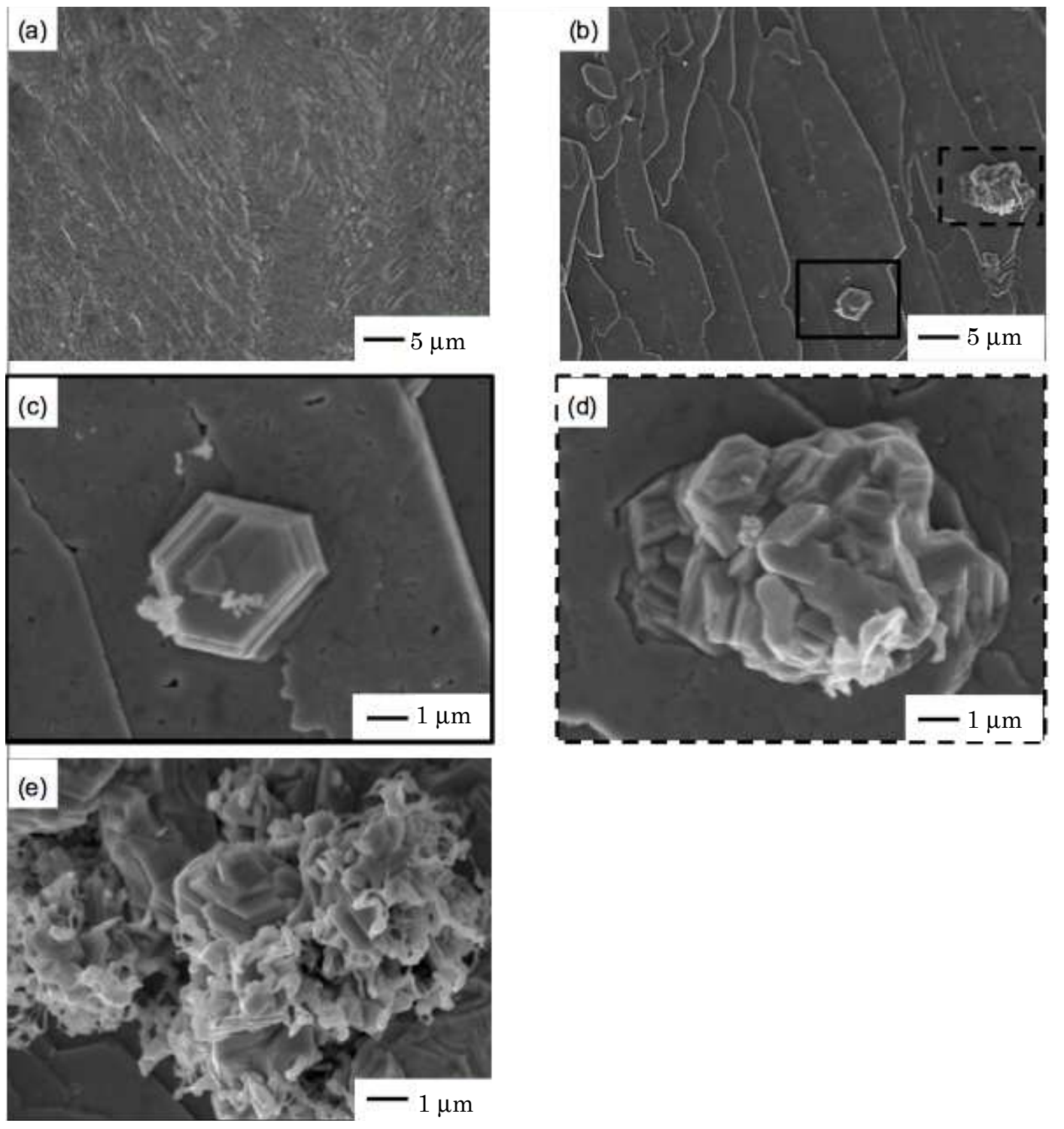

Fig. 7. SEM images of the working electrode. (a) polycrystalline case (Test \#9); (b)-(e) (0001) case (Test \#14). The current density is $10 \mathrm{~mA} \mathrm{~cm}^{-2}$ and the charge capacity is $1.0 \mathrm{mAh} \mathrm{cm}^{-2}$. 

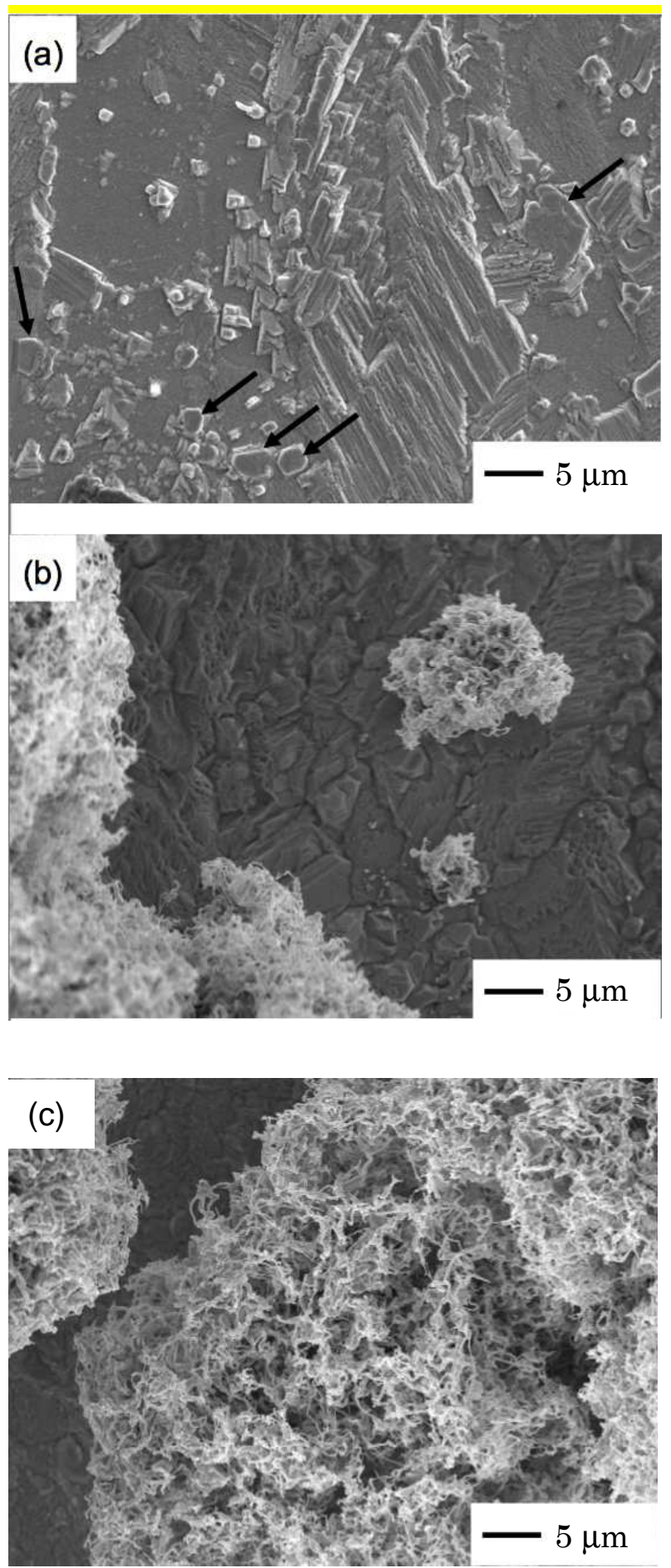

Fig. 8. SEM images of the zinc anode in the polycrystalline case. The current density is $10 \mathrm{~mA} \mathrm{~cm}^{-2}$. The charge capacity is (a) $5.0 \mathrm{mAh} \mathrm{cm}^{-2}$ (Test \#10) and (b) $7.5 \mathrm{mAh} \mathrm{cm}^{-2}$ (Test \#11), and (c) $10.0 \mathrm{mAh} \mathrm{cm}^{-2}$ (Test \#12), respectively. 
(a) smooth terrace and step

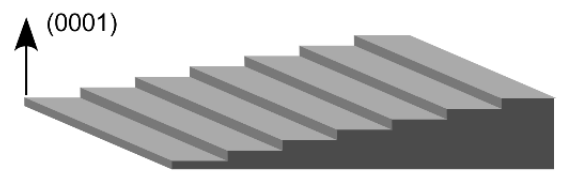

(b) wider terrace and bunched step

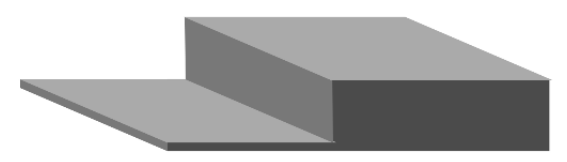

(c) island structure formation via repeated $2 \mathrm{D}$-nucleation $\prod$ current concentration

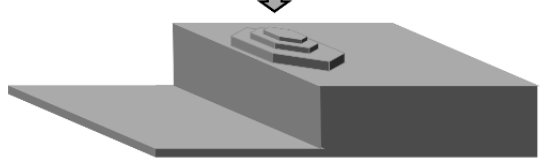

(d) non-uniform electrodeposition on island structure

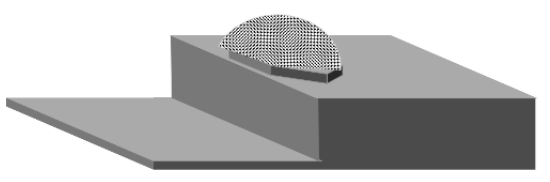

Fig. 9. Formation process of non-uniform electrodeposition in the (0001) case: (a) At the early stage of charge, layer-by-layer growth proceeds with smooth surface while maintaining step-terrace structure; (b) wide terraces are formed due to step bunching; (c) spotty structures form via recurring 2D-nucleations on the wide terraces and the current concentration at the ledges results in the preferential formation of electrodeposition; and (d) the electrodeposition enhance more precipitation, and finally, the porous non-uniform electrodeposition forms. 